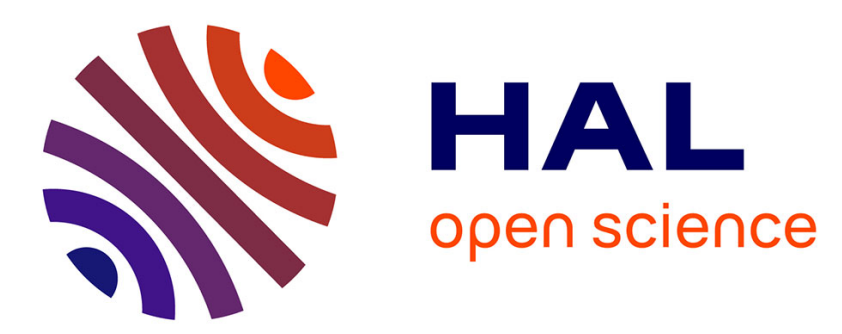

\title{
Complex continuous nonlinear systems: Their back box identification and their control
}

\author{
Michel Fliess, Cédric Join, Hebertt Sira-Ramirez
}

\section{To cite this version:}

Michel Fliess, Cédric Join, Hebertt Sira-Ramirez. Complex continuous nonlinear systems: Their back box identification and their control. 14th IFAC Symposium on System Identification, SYSID 2006, Mar 2006, Newcastle, Australia. inria-00000824

\section{HAL Id: inria-00000824 \\ https://hal.inria.fr/inria-00000824}

Submitted on 22 Nov 2005

HAL is a multi-disciplinary open access archive for the deposit and dissemination of scientific research documents, whether they are published or not. The documents may come from teaching and research institutions in France or abroad, or from public or private research centers.
L'archive ouverte pluridisciplinaire $\mathbf{H A L}$, est destinée au dépôt et à la diffusion de documents scientifiques de niveau recherche, publiés ou non, émanant des établissements d'enseignement et de recherche français ou étrangers, des laboratoires publics ou privés. 


\title{
COMPLEX CONTINUOUS NONLINEAR SYSTEMS: THEIR BLACK BOX IDENTIFICATION AND THEIR CONTROL
}

\author{
Michel Fliess* Cédric Join ** \\ Hebertt Sira-Ramírez ${ }^{* * *}$ \\ * Projet ALIEN, INRIA Futurs 83 \\ Équipe MAX, LIX (UMR-CNRS 7161) \\ École polytechnique, 91128 Palaiseau, France \\ E-mail: Michel.Fliess@polytechnique.fr \\ ** Projet ALIEN, INRIA Futurs \& \\ CRAN (UMR-CNRS 7039), Université Henri Poincaré \\ (Nancy I), BP 239, 54506 Vandouvre-lès-Nancy, France \\ E-mail: cedric.join@cran.uhp-nancy.fr \\ *** CINVESTAV-IPN, Sección de Mecatrónica, \\ Departamento de Ingeniería Eléctrica, Avenida IPN, No. \\ 2508, Col. San Pedro Zacatenco, AP 14740 \\ 07300 México D.F., México \\ E-mail: hsira@mail.cinvestav.mx
}

\begin{abstract}
Recent advances in estimation theory permit a new approach to nonlinear black box identification, where a phenomenological model is replacing a precise mathematical description. Convincing simulations are provided for two examples:

- the classic ball and beam system,

- a large scale linear system, where our setting may regarded as a powerful alternative to model reduction. Copyright (C)2006 IFAC
\end{abstract}

Keywords: Nonlinear systems, black box identification, model reduction, derivatives of a noisy signal, input-output representation, differential algebra.

\section{INTRODUCTION}

The great industrial popularity of PID controllers (see, e.g., (Aström et al., 1995; O'Dwyer, 2003)) is not only due to their conceptual simplicity but also to the fact that practitioners do not employ any precise mathematical model of the plant. Nevertheless the quite delicate tuning of PID controllers, their poor performance with systems of high dimensions and/or with severe nonlinearities have prompted the introduction of new standpoints, like fuzzy logic and neural nets, which do not seem to have encountered the same success.
This communication is devoted to a new approach on black box identification of complex continuoustime nonlinear systems. We do not try anymore to obtain an accurate mathematical model and we replace this quite difficult task in the single-input single-output case by a phenomenological ${ }^{1}$ model, i.e., an ordinary differential equation

$$
y^{(n)}=F+\alpha u+\beta
$$

where

\footnotetext{
1 This word refers of course to Husserl's philosophy (see, e.g., (Bernet et al., 1996)).
} 
- $n \geq 1$ and, most often, $n=1$, or 2 ,

- $\alpha, \beta \in \mathbb{R}$ are "non-physical" constant parameters which are tuned by the practitioner,

- $F$ is given thanks to the knowledge of $y^{(n)}$, $u, \alpha, \beta$.

If $n=1,2$, the desired behavior is obtained by an elementary PID controller of the form

$$
u \frac{1}{\alpha}\left(y_{*}^{(n)}-F-\beta+K_{P} e+K_{I} \int e+K_{D} \dot{e}\right)
$$

where $y_{*}$ is the reference trajectory, $e=y-y_{*}$, $K_{P}, K_{I}, K_{D} \in \mathbb{R}$ are suitable gains. Note that the tuning of the PID gains is quite straightforward.

The key tool for obtaining equation (1) is the possibility of estimating derivatives of a noisy signal, here the output signal $y$. This has been achieved in (Fliess and Sira-Ramírez, 2004), where efficient state reconstructors have been obtained which permit state feedbacks.

Lack of space is imposing us illustrations where the model equations are already known. We have chosen a rather difficult nonlinear system and a linear system of quite large dimension. Numerical simulations in both cases may be favorably compared with existing techniques using model equations.

Section 2 is devoted to a short survey of nonlinear systems via differential algebra. The analysis of the derivative estimation of a noisy signal is summarized in section 3 . The basic principles for the computer implementation of our black box identification scheme are reviewed in section 4 . Section 5 is devoted to the two illustrative case-studies. A short conclusion describes some forthcoming works.

\section{REVIEW OF SYSTEM THEORY}

\subsection{Differential fields}

A differential field ${ }^{2} \mathfrak{K}$ is a commutative field $^{3}$ which is equipped with a derivative $\frac{d}{d t}$, i.e., a mapping $\mathfrak{K} \rightarrow \mathfrak{K}$ such that, $\forall a, b \in \mathfrak{K}$,

- $\frac{d}{d t}(a+b)=\dot{a}+\dot{b}$,

- $\frac{d}{d t}(a b)=\dot{a} b+a \dot{b}$.

A constant $c \in \mathfrak{K}$ is an element such that $\dot{c}=0$. The set of all constants is the subfield of constants.

Consider the differential field extension $\mathfrak{L} / \mathfrak{K}$, i.e., two differential fields $\mathfrak{K}, \mathfrak{L}$ such that

- $\mathfrak{K} \subseteq \mathfrak{L}$,

2 See (Chambert-Loir, 2005; Kolchin, 1973) for more details.

3 All the fields are assumed to be of characteristic zero. See (Chambert-Loir, 2005) for basics on field theory.
- the derivative of $\mathfrak{K}$ is equal to the restriction to $\mathfrak{K}$ of the derivative of $\mathfrak{L}$.

Write $\mathfrak{K}\langle S\rangle, S \subset \mathfrak{L}$, the differential subfield of $\mathfrak{L}$ generated by $\mathfrak{K}$ and $S$. Assume that $\mathfrak{L} / \mathfrak{K}$ is finitely generated, i.e., $\mathfrak{L}=\mathfrak{K}\langle S\rangle$, where $S$ is finite. An element $\xi \in \mathfrak{L}$ is said to be differentially algebraic over $\mathfrak{K}$ if, and only if, it satisfies an algebraic differential equation, i.e., $P\left(\xi, \ldots, \xi^{(n)}\right)=0$ where $P$ is a polynomial over $\mathfrak{K}$ in $n+1$ indeterminates. The extension $\mathfrak{L} / \mathfrak{K}$ is said to be differentially algebraic if, and only if, any element of $\mathfrak{L}$ is differentially algebraic over $\mathfrak{K}$. The following result plays an important rôle: The extension $\mathfrak{L} / \mathfrak{K}$ is differentially algebraic if, and only if, its transcendence degree is finite.

An element of $\mathfrak{L}$ which is not differentially algebraic over $\mathfrak{K}$ is said to be differentially transcendental over $\mathfrak{K}$. An extension $\mathfrak{L} / \mathfrak{K}$ which is not differentially algebraic is said to be differentially transcendental. A set $\left\{\xi_{\iota} \in \mathfrak{L} \mid \iota \in I\right\}$ is said to be differentially algebraically independent over $\mathfrak{K}$ if, and only if, they are not related by any nontrivial algebraic differential relation over $\mathfrak{K}$, i.e., $Q\left(\xi_{\iota}^{\left(\nu_{\iota}\right)}\right)=0$, where $Q$ is a polynomial over $\mathfrak{K}$ in several indeterminates, implies $Q \equiv 0$. Two maximal sets of differentially algebraically independent elements have the same cardinality, i.e., the same number of elements, which is the differential transcendence degree of the extension $\mathfrak{L} / \mathfrak{K}$. Any such set is called a differential transcendence basis.

\subsection{Nonlinear control systems}

Let $k$ be a given ground differential field. A sys$t e m^{4}$ is a finitely generated differentially transcendental extension $K / k$. Let $m$ be its differential transcendence degree. A set of (independent) control variables $\mathbf{u}=\left(u_{1}, \ldots, u_{m}\right)$ is a differential transcendence basis of $K / k$. It implies that the extension $K / k\langle\mathbf{u}\rangle$ is differentially algebraic. A set of output variables $\mathbf{y}=\left(y_{1}, \ldots, y_{p}\right)$ is a subset of K.

Let $\mathbf{x}=\left(x_{1}, \ldots, x_{n}\right)$ be a transcendence basis of $K / k\langle\mathbf{u}\rangle$, where $n$ is its transcendence degree. It yields the generalized state-variable representation:

$$
\begin{aligned}
& A_{i}\left(\dot{x}_{i}, \mathbf{x}, \mathbf{u}, \ldots, \mathbf{u}^{(\alpha)}\right)=0 \\
& B_{j}\left(y_{j}, \mathbf{x}, \mathbf{u}, \ldots, \mathbf{u}^{(\beta)}\right)=0
\end{aligned}
$$

where $A_{i}, i=1, \ldots, n, B_{j}, j=1, \ldots, p$, are polynomials over $k$.

Consider the SISO case, i.e., $m=p=1$. Let $u$, $y$ be the control and output variables. The same

\footnotetext{
4 See (Fliess et al., 1995; Rudolph, 2003; Sira-Ramírez and Agrawal, 2004) for more details.
} 
reasoning as above yields the following inputoutput representation

$$
\Phi\left(y, \ldots, y^{(\bar{N})}, u, \ldots, u^{(\bar{M})}\right) 0
$$

where $\Phi$ is a polynomial over $k$.

Remark 2.1. Assume that $y$ is given. If $k=\mathbb{R}$ the qualitative behavior of equation (3), when viewed as a differential equation with respect to the unknown $u$, permits to define minimum and nonminimum phase systems (see also (Isidori, 1999)).

\section{DERIVATIVES OF NOISY SIGNALS}

Consider a real-valued time function $x(t)$ which is assumed to be analytic on some interval $t_{1} \leq$ $t \leq t_{2}$. Assume for simplicity's sake that $x(t)$ is analytic around $t=0$ and introduce its truncated Taylor expansion

$$
x(t)=\sum_{\nu=0}^{N} x^{(\nu)}(0) \frac{t^{\nu}}{\nu !}+o\left(t^{N}\right)
$$

Approximate $x(t)$ in the interval $(0, \varepsilon), \varepsilon>0$, by a polynomial $x_{N}(t)=\sum_{\nu=0}^{N} x^{(\nu)}(0) \frac{t^{\nu}}{\nu !}$ of degree $N$. The usual rules of symbolic calculus in Schwartz's distributions theory yield

$x_{N}^{(N+1)}(t)=x(0) \delta^{(N)}+\dot{x}(0) \delta^{(N-1)}+\cdots+x^{(N)}(0) \delta$

where $\delta$ is the Dirac measure at 0 . From $t \delta=$ $0, t \delta^{(\alpha)}=-\alpha \delta^{(\alpha-1)}, \alpha \geq 1$, we obtain the following triangular system of linear equations for determining estimated values $\left[x^{(\nu)}(0)\right]_{e}$ of the derivatives ${ }^{5} x^{(\nu)}(0)$ :

$$
\begin{aligned}
& t^{\alpha} x^{(N+1)}(t)=t^{\alpha}\left([x(0)]_{e} \delta^{(N)}\right. \\
& \left.+[\dot{x}(0)]_{e} \delta^{(N-1)} q+\cdots+\left[x^{(N)}(0)\right]_{e} \delta\right) \\
& \alpha=0, \ldots, N
\end{aligned}
$$

The time derivatives of $x(t)$ and the Dirac measures and its derivatives are removed by integrating with respect to time both sides of equation (4) at least $N$ times:

$$
\begin{aligned}
& \int^{(\nu)} \tau_{1}^{\alpha} x^{(N+1)}\left(\tau_{1}\right)=\int^{(\nu)} \tau_{1}^{\alpha}\left([x(0)]_{e} \delta^{(N)}\right. \\
& \left.+[\dot{x}(0)]_{e} \delta^{(N-1)}+\cdots+\left[x^{(N)}(0)\right]_{e} \delta\right) \\
& \nu \geq N, \alpha=0, \ldots, N
\end{aligned}
$$

where $\int^{(\nu)}=\int_{0}^{t} \int_{0}^{\tau_{\nu-1}} \ldots \int_{0}^{\tau_{1}}$ is an iterated integral. A quite accurate value of the estimates may be obtained with a small time window $[0, t]$.

Remark 3.1. Those iterated integrals are moreover low pass filters ${ }^{6}$. They are attenuating highly fluctuating noises, which are usually dealt with in a statistical setting. We therefore do not need

\footnotetext{
5 Those quantities are linearly identifiable (Fliess and Sira-Ramírez, 2003).

6 Those iterated integrals may be replaced by more general low pass filters, which are defined by strictly proper rational transfer functions.
}

any knowledge of the statistical properties of the noises.

Remark 3.2. See (Reger et al., 2005; Fliess et al., 2006) for further details on the numerical implementation.

Remark 3.3. See, e.g., (Fliess and Sira-Ramírez, 2004; Fliess et al., 2005b; Reger et al., 2005) for various applications to nonlinear systems (state and parametric estimations, fault-diagnosis and fault-tolerant control).

Remark 3.4. See, e.g., (Fliess et al., 2004; Fliess et al., 2005a) for applications to signal processing.

Remark 3.5. Any other real-time technique for estimating the derivatives of a noisy signal could be adopted.

\section{BLACK BOX IDENTIFICATION}

As in flatness-based control ${ }^{7}$ a reference trajectory is selected for the output $y$ (see, e.g., (Fliess et al., 1995; Rudolph, 2003; Sira-Ramírez and Agrawal, 2004)). The parameters $\alpha$ and $\beta$ in equation (1) are chosen such that

(1) the magnitude of the control variable $u$ is suitable,

(2) $\beta \neq 0$ if $u$ does not appear linearly in equation (3). i.e.,

$$
\frac{\partial \Phi}{\partial u}(u=0) \equiv 0
$$

The two following steps are needed in order to avoid algebraic loops:

(1) The selected integer $n$ in equation (1) is related to equation (3) by

$$
\frac{\partial \Phi}{\partial y^{(n)}} \not \equiv 0
$$

(2) The value of $F$, which is equal $y^{(n)}-\alpha u-\beta$, is given via a discretisation procedure of the form

$$
F_{\kappa}=\left[y_{\kappa}^{(n)}\right]_{e}-\alpha u_{\kappa-1}-\beta
$$

where $\left[\boldsymbol{\bullet}_{\kappa}\right] e$ is designating the estimate at time $\kappa$.

Remark 4.1. Our procedure might lead for nonminimum phase systems to divergent values of $u$ when time $t$ is increasing and therefore to large values of $F$.

\footnotetext{
7 Equation (1) defines indeed a flat system if we consider the function $F$ as only time dependant. There exists more generally some similarity with techniques stemming from inversion-based feedforward control (see, e.g., (Graichen et $a l ., 2005)$ and the references therein.).
} 
Remark 4.2. For most minimum phase systems arising in practice, we might choose $n=1$, or 2. We are therefore approaching a kind of "universal" controller, where the tuning difficulties of classic PID control are largely overcome.

\section{TWO EXAMPLES}

The model equations of the two examples below are only utilized for providing the reference controls in the numerical simulations.

\subsection{The ball and beam system}

5.1.1. Model description This well known nonlinear system, which has been studied via various techniques (see, e.g., (Sastry, 1999; Zhang et al., 2002)), is not linearizable by static state feedback and therefore not flat (see, e.g., (SiraRamírez and Agrawal, 2004)) and quite difficult to control. It obeys the equation ${ }^{8}$

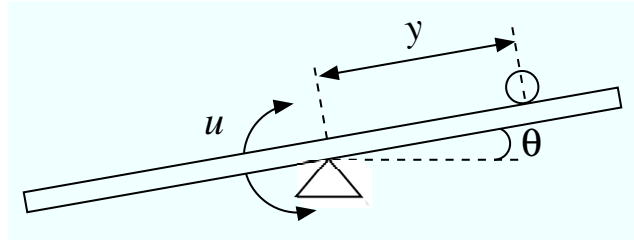

Fig. 1. The ball and beam system

$$
\ddot{y}=B y \dot{u}^{2}-B G \sin u
$$

where the control variable $u=\theta$ is the angle of the beam and the measured output

$$
y_{m}=y+\varpi
$$

is corrupted by some noise $\varpi$. The parameters $B=0.71, G=9.81$ are constant.

5.1.2. Simulation results We have chosen $n=$ $2, \alpha=100, \beta=0$. For our numerical experiments, the magnitude of the control variable $u$ and of its derivative are bounded: $-\pi / 3<u<\pi / 3$, $-\pi<\dot{u}<\pi$. For the two types of reference trajectories (a Bézier polynomial and a sinusoidal function in figures 2 and 3), the system output shows a very good behavior. A PID control of type (2) has been utilized. Figures 2-(b), 3-(b), 2-(c), 3 -(c) present respectively the control input and the estimation of $F$ in noise-free case. Trajectory tracking is to compare with figures $2-(\mathrm{d}), 3-(\mathrm{d})$ in the noisy case ( $\varpi$ in equation (6) is zero-mean and Gaussian of magnitude more than 1\%). The performance remains quite acceptable.

8 Note that the equation below, which contains a sine function, is not differentially algebraic and therefore not of the kind of those considered in section 2. This slight difficulty may be easily overcome by utilizing $\tan \frac{u}{2}$ (see (Fliess et al., 1995)).

\subsection{Linear example}

5.2.1. Model description The quite large linear system

$$
\frac{y(s)}{u(s)}=\frac{s^{5}}{\prod_{i=1}^{6}\left(s+p_{i}\right)}
$$

where $p_{1}=-1, p_{2}=-0.1, p_{3}=-0.01, p_{4}=$ $0.05, p_{5}=0.5, p_{6}=5$, which exhibits slow and fast poles, might be usually treated via modelreduction techniques (see, e.g., (Antoulas, 2005; Obinata and Anderson, 2001)).

5.2.2. Numerical simulations Here $n=1$. We are therefore utilizing around the reference trajectory a PI controller, i.e., a controller of type (2) where $K_{D}=0$. The added noise is the same as in section 5.1.2. The efficiency of our method is clearly demonstrated by figure 4 : the trajectory tracking is nearly perfect.

\section{CONCLUSION}

Our approach, which could be also characterized as "non-model-based predictive control", is extended to multivariable systems in (Fliess et al., 2006). The case of non-minimum phase systems will be investigated in further studies.

Forthcoming publications will

- make our approach with respect to universal controllers more precise,

- describe how our black box identification may be easily applied to concrete industrial plants, where a parametric identification is difficult to achieve.

\section{REFERENCES}

A. C. Antoulas, 2005, Approximation of Large-Scale Dynamical Systems, SIAM, Philadelphia.

K.J. Aström, T. Hägglund, 1995, PID Controllers: Theory, Design and Tuning, $2^{\text {nd }}$ ed., Instrument Soc. Amer., Research Triangle Park, NC.

R. Bernet, I. Kern, E. Marbach, 1996, Edmund Husserl. Darstellung seines Denkens, 2. Auflage, Felix Meiner Verlag, Hamburg.

A. Chambert-Loir, 2005, A Field Guide to Algebra, Springer, Berlin.

A. O'Dwyer, 2003, Handbook of PI and PID Controller Tuning Rules, Imperial College Press, London.

M. Fliess, C. Join, M. Mboup, H. Sira-Ramírez, 2004, Compression différentielle de transitoires bruités, C.R. Acad. Sci. Paris Ser. I, 339, 821-826.

M. Fliess, C. Join, M. Mboup, H. Sira-Ramírez, 2005a, Analyse et représentation de signaux transitoires: application à la compression, au débruitage et à la détection de ruptures, Actes Coll. GRETSI, Louvainla-Neuve.

M. Fliess, C. Join, M. Mboup, H. Sira-Ramírez, 2006, Vers une commande multivariable sans modèle, Actes Coll. Internat. Francoph. Automat. (CIFA), Bordeaux. 


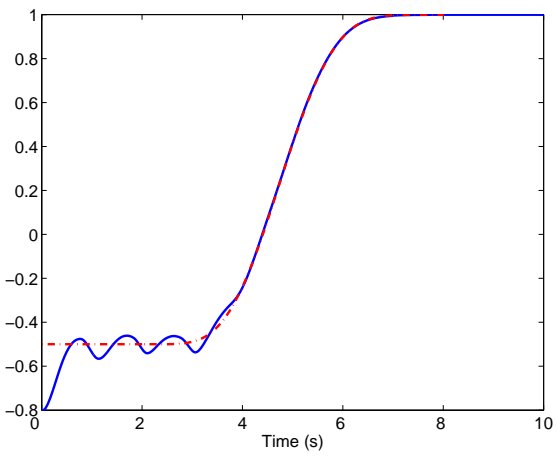

(a) Reference (- -) and actual output

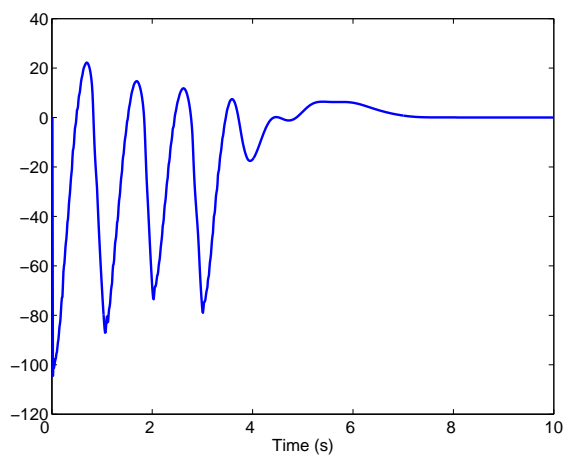

(c) Estimation of $F$

Fig. 2. Polynomial trajectory

M. Fliess, C. Join, H. Sira-Ramírez, 2005b, Closed-loop fault-tolerant control for uncertain nonlinear systems, in Control and Observer Design for Nonlinear Finite and Infinite Dimensional Systems, T. Meurer, K. Graichen, E.D. Gilles (Eds), Lect. Notes Control Informat. Sci. 322, Springer, Berlin, pp. 217-233.

M. Fliess, J. Lévine, P. Martin, P. Rouchon, 1995, Flatness and defect of non-linear systems: introductory theory and examples, Internat. J. Control, 61, 1327-1361.

M. Fliess, H. Sira-Ramírez, 2003, An algebraic framework for linear identification, ESAIM Control Optim. Calc. Variat., 9, 151-168.

M. Fliess, H. Sira-Ramírez, 2004, Control via state estimations of some nonlinear systems, Proc. Symp. Nonlinear Control Systems (NOLCOS), Stuttgart.

K. Graichen, V. Hagenmeyer, M. Zeitz, 2005, A new approach to inversion-based feedforward control design for nonlinear systems, Automatica, 41, 2033-2041.

A. Isidori, 1999, Nonlinear Control Systems II, Springer, Berlin.

E.R. Kolchin, 1973, Differential Algebra and Algebraic Groups, Academic Press, New York.

G. Obinata, B.D.O. Anderson, 2001, Model Reduction for Control Systems, Springer, Berlin.

J. Reger, H. Sira-Ramírez, M. Fliess, 2005, On nonasymptotic observation of nonlinear systems, Proc. $C D C$-ECC'05, Seville.

J. Rudolph, 2003, Beiträge zur flacheitsbasierten Folgeregelung linearer und nichtlinearer Syteme endlicher und undendlicher Dimension, Shaker Verlag, Aachen.

S. Sastry, 1999, Nonlinear Systems, Springer, Berlin.

H. Sira-Ramírez, S. Agrawal, 2004, Differentially Flat Systems, Marcel Dekker, New York.

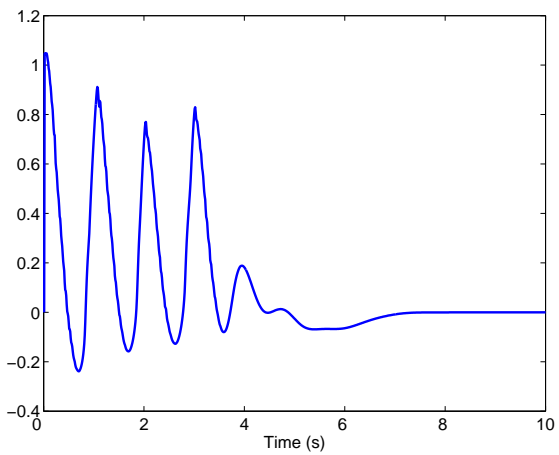

(b) Input control

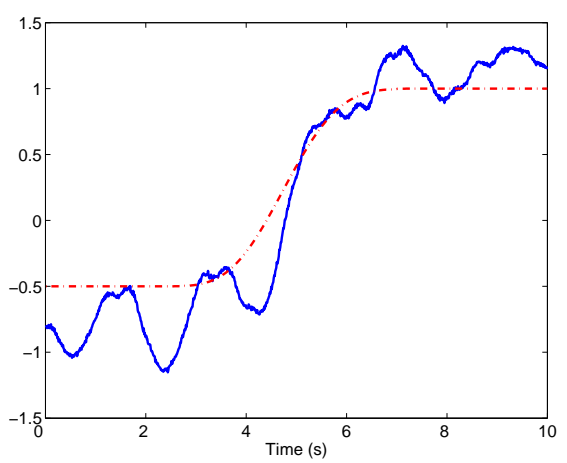

(d) Reference (- -) and actual output in the noisy case

Y. Zhang, D. Jiang, J. Wang, A recurrent neural network for solving Sylvester equation with time-varying coefficients, IEEE Trans. Neural Networks, 13, 10531063. 


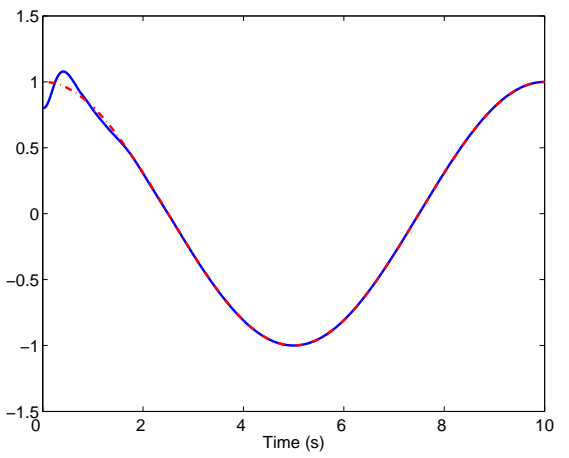

(a) Reference (- -) and actual output

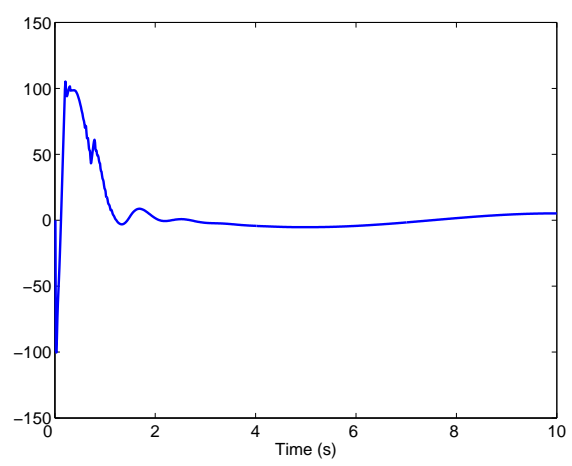

(c) Estimation of $F$

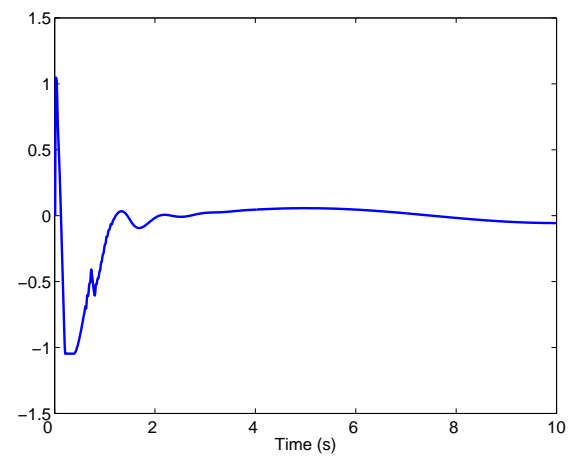

(b) Input control

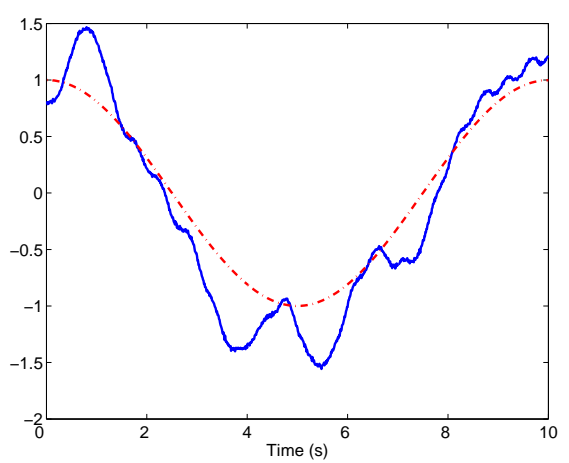

(d) Reference (- -) and actual output in the noisy case

Fig. 3. Sinusoidal trajectory

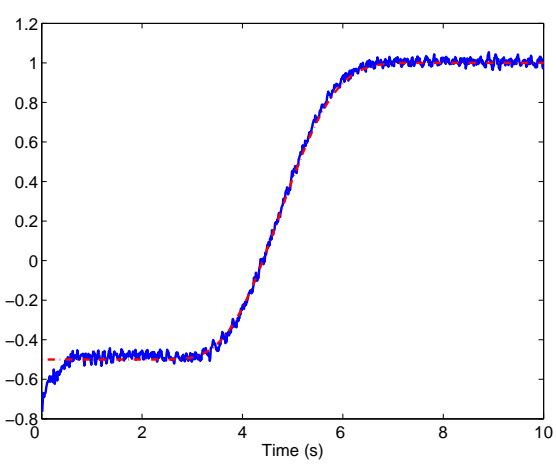

(a) Reference (- -) and output

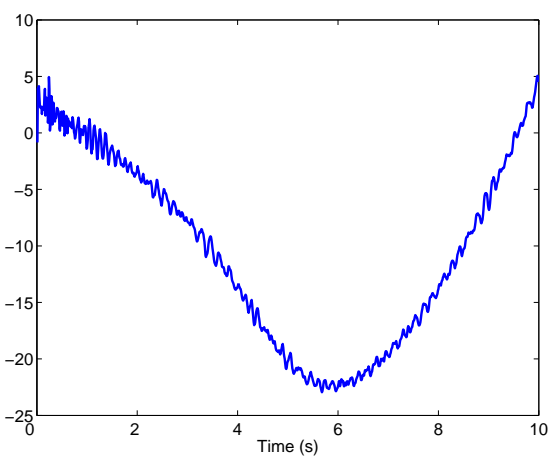

(c) Estimation of $F$

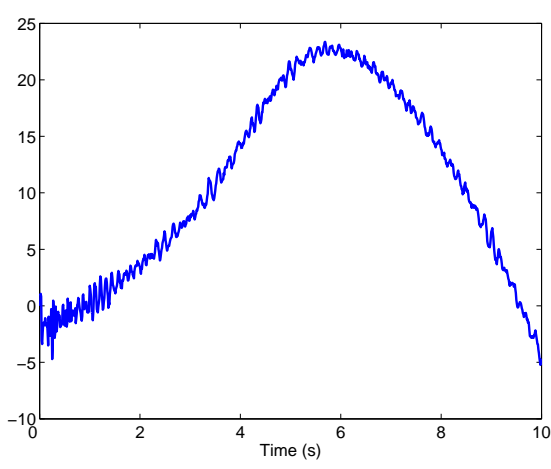

(b) Input control

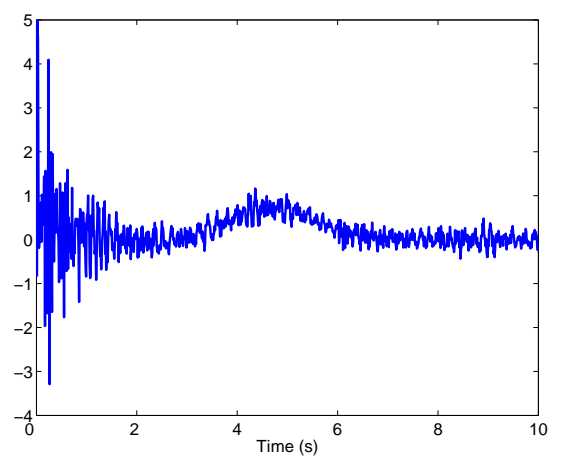

(d) Estimation of the first output derivative

Fig. 4. The linear system 